\title{
Another triumph of hope over experience?
}

\author{
REVISITING... TREATMENT OF THE PATIENT WITH LONG-TERM SCHIZOPHRENIA
}

\section{Ann M. Mortimer}

\begin{abstract}
Schizophrenia continues to challenge services: recent advances in antipsychotic drug treatment and psychosocial interventions are hindered by non-adherence, disengagement and substance misuse. Furthermore, new side-effect concerns attach to atypical drugs, and psychosocial interventions may be underresourced or of unproven benefit. It is important to address all issues with which patients, families and carers need assistance, and to take a well-informed, creative approach to pharmacological treatment, using medication according to individual patient need rather than mechanistic adherence to guidelines. Psychiatrists should be realistic in their expectations of patient outcome, accepting outcomes that fall short of recovery and imply long-term supportive care, and insisting that this care be available to their patients. Psychiatrists should support early diagnosis and intervention as being possibly the only means to alleviate the burden of long-term schizophrenia for patients, families and services.
\end{abstract}

This article continues a series revisiting early contributions to APT (see also Cowen, 2005; Edwards, 2005; Gournay, 2005; Seivewright et al, 2005, this issue). The original article is available on our website (http://apt.rcpsych.org), as a data supplement to the online version of the present article. Readers might also be interested in contributions to APT by Kerwin \& Bolonna (2005) on clozapineresistant schizophrenia and Singh \& Fisher (2005) on early intervention in psychosis.

Although some patients with first-episode schizophrenia achieve sustained symptomatic and functional recovery, the overall rate of recovery during the early years of the illness is low: $13.7 \%$ at 2 years in a recent large study (Robinson et al, 2004). Progressive deterioration subsequent to repeated relapses makes a long-term perspective on treatment essential. As mentioned in my earlier article (Mortimer, 1997), two objectives are crucial here. The first is prevention of relapses, most of which are caused by non-adherence to treatment regimens (Davis, 1975) and/or substance misuse (Hunt et al, 2002). Currently, about half of patients are nonadherent (Lacro et al, 2002) and half misuse substances (Swafford et al, 2000): there is significant association between these behaviours (Margolese et al, 2002).

The second objective is the management of unresolved symptoms and social deficits. A wide range of approaches are available, including sophisticated use of medication, psychotherapy, and social and family support. Individuals who cannot be returned to adequate function will need ongoing support and care, which is best achieved within a multidisciplinary framework such as the care programme approach (CPA).

\section{Relapse: prevention and treatment}

Without medication, for each month after an acute episode a further $8-16 \%$ of patients relapse (Davis \& Casper, 1977). Hunt et al (2002) found that the median time to relapse in substance-misusing patients who failed to adhere to medication regimens was 5 months. The reasons for non-adherence remain unchanged: loss of insight is probably a substantial contributor, added to which are issues surrounding the medication such as side-effects and expense, and factors that could be addressed by greater understanding of the illness (Box 1).

Ideally, the patient and family will have had the benefit of a first-episode service offering family work, cognitive-behavioural therapy and psychoeducation about schizophrenia and its treatment.

Ann Mortimer became Foundation Chair in Psychiatry at the University of Hull in 1996 (Hertford Building, Cottingham Road, Hull HU6 7RX, UK. Tel: 01482 464565; fax: 01482 464569; e-mail: a.m.mortimer@hull.ac.uk), moving from Imperial College London. Professor Mortimer runs assertive outreach for Hull, and rehabilitation services for Hull and East Yorkshire. Her research interests reflect her clinical interests, with a focus on the pharmacological treatment of severe mental illness; she is in addition the lead consultant for maternal mental health in Hull and East Yorkshire, and Deputy Chief Examiner to the Royal College of Psychiatrists. Professor Mortimer has received educational and research funding from all the pharmaceutical companies that produce atypical antipsychotic drugs. These include Novartis, Janssen-Cilag, AstraZeneca, Sanofi Synthelabo, Eli Lilly and Bristol Myers Squibb. 
Box 1 Barriers to optimal outcomes in longterm treatment

- Non-adherence

- Substance misuse

- Lack of psychosocial interventions

- Treatment-resistant positive and negative symptoms

- Cognitive impairment

- Social exclusion

Unfortunately, most existing patients will have 'missed the boat' in this regard. Services should make provision for ongoing interventions of this nature, with a focus on relapse prevention. This would include medication management and adherence work, the recognition of links between stressful events and exacerbation of illness, identification of the characteristic 'relapse signature', and addressing substance misuse. The success of such approaches rests on a constructive relationship between professionals, patient and family. Every effort should be made to resolve difficulties: to disabuse the patient and family of misapprehensions about schizophrenia, to clear up past and current misunderstandings regarding the input of services, to give an honest and intelligible appraisal of the patient's condition without being judgemental, and to support the patient and family in coming to terms with the diagnosis and its implications. There is a strong evidence base for family interventions: a fairly recent meta-analysis concluded that family therapy had clear preventive effects on the outcomes of psychotic relapse and readmission, in addition to benefits for medication adherence (Pilling et al, 2002a).

Regarding drug treatment, the National Institute for Clinical Excellence (NICE) guidance suggests that an atypical antipsychotic is appropriate for patients who have relapsed or who experience unacceptable side-effects on conventional antipsychotic drugs (National Institute for Clinical Excellence, 2002). Patients failing to respond adequately to two antipsychotics, one an atypical, given sequentially in therapeutic doses for 6-8 weeks, should be considered for clozapine treatment. Depot medication should be used when appropriate: long-acting risperidone was not available when the NICE guidance was published.

Essentially, acute treatment in relapse involves a combination of symptom control with effective medication, and psychosocial interventions aimed at obviating relapse in the future. For patients nearing discharge, a process of gradually increasing responsibility for medication should occur. Ideally, they will have full responsibility prior to discharge. Once the acute psychosis is reasonably well controlled, the objectives and process of treatment very much merge into those of longer-term maintenance.

\section{Atypical antipsychotics}

The most commonly used atypicals are listed in Box 2 and discussed briefly below.

\section{Risperidone}

Risperidone is superior to conventional antipsychotics for positive and negative symptoms (Song, 1997). At a daily dose of $6 \mathrm{mg}$, it causes no more extrapyramidal symptoms (EPS) than placebo (Marder \& Meibach, 1994). It does, however, cause hyperprolactinaemia. Other side-effects include orthostatic hypotension, sedation, sexual dysfunction and weight gain. In clinical practice many individuals, even during acute relapse, can be managed on doses lower than $6 \mathrm{mg}$ (Lane et al, 2000).

Risperidone is available in a sustained-release intramuscular formulation of microspheres for 2weekly administration. In a 3-month, double-blind placebo-controlled randomised trial with 400 individuals, this formulation proved superior to placebo, with no more withdrawals from the trial because of side-effects than occurred with those on placebo (Green, 2000).

\section{Olanzapine}

Olanzapine is superior to conventional antipsychotics for positive symptoms (Leucht et al, 1999), and is much superior to conventional treatment for negative symptoms (Kopelowicz et al, 2000). Its most common side-effects are sedation, dizziness and weight gain. Extrapyramidal symptoms are unusual, apart from occasional akathisia. Olanzapine does not induce hyperprolactinaemia to a sustained or clinically significant degree. Cardiac safety appears highly satisfactory (Czekalla et al, 2001).

Box 2 Currently available atypical antipsychotics

- Risperidone

- Olanzapine

- Quetiapine

- Zotepine

- Amisulpride

- Aripiprazole

- Clozapine 


\section{Quetiapine}

Quetiapine is at least as effective as haloperidol for positive symptoms (Arvanitis et al, 1997). However, evidence for better efficacy in negative symptoms is more equivocal (Peuskens \& Link, 1997; Leucht et al, 1999). The outstanding advantages of quetiapine are its placebo-level induction of EPS across the dosage range and that it does not affect prolactin levels. Typically, most clinicians find maximal doses necessary in patients with chronic schizophrenia. Side-effects are infrequent and include somnolence, dry mouth, abdominal pain and weight gain. Overall, quetiapine is possibly the best tolerated antipsychotic, either conventional or atypical (comparisons with aripiprazole in this regard are not available).

\section{Zotepine}

Zotepine is as effective as haloperidol in the treatment of positive symptoms (Raniwalla et al, 1996) and is at least as effective for negative symptoms (Barnas et $a l, 1992)$. Side-effects of zotepine include insomnia, somnolence, anxiety, headache, constipation, weight gain, dyspepsia and dry mouth. The incidence of EPS is lower than with conventional drugs, although weight gain and tachycardia may be more of a problem (Hwang et al, 2001).

\section{Amisulpride}

Amisulpride is at least as effective as conventional treatment for positive symptoms and low doses may substantially reduce negative symptoms (Moller, 2000). It induces far fewer EPS than haloperidol, with placebo levels up to the dose of $300 \mathrm{mg}$ daily. It does, however, cause hyperprolactinaemia. Other sideeffects are uncommon, but can include agitation, anxiety and insomnia.

\section{Aripiprazole}

Aripiprazole is a partial dopamine agonist that upgrades or downgrades dopaminergic neurotransmission, depending on the level of endogenous dopamine in the synapse. There is experimental evidence for regional imbalances in endogenous dopamine levels in schizophrenia, with an excess in mesolimbic areas and a deficit in mesocortical areas (Abi-Dargham, 2002). It is also a $5-\mathrm{HT}_{1 \mathrm{~A}}$ agonist, thought to confer anxiolytic and antidepressant properties as well as release frontal dopamine. Aripiprazole is at least as effective as conventional antipsychotic drugs in both acute and maintenance treatment, and has placebo-level EPS. It appears to be an exceptionally well-tolerated antipsychotic (Bowles \& Levin, 2003). The most common side-effects are headache, anxiety, insomnia and nausea: these seem to be mild and transient, and not to constitute a reason for discontinuation. There is no known association with weight gain or QTc prolongation, and no hyperprolactinaemia. Unfortunately, aripiprazole is not generally effective in treatment-resistant schizophrenia.

\section{Clozapine}

Clozapine is the only antipsychotic indicated for treatment-resistant schizophrenia. Unfortunately, its side-effect profile, although virtually free of EPS, includes neutropenia in 1 in 43 patients (Munro et al, 1999), weight gain, sedation, hypersalivation, myoclonus and type II diabetes. Despite these drawbacks, and the requirement for regular monitoring of white cell count, clozapine can be highly effective in controlling the severe symptoms of individuals able to tolerate it. The NICE guidance recommends a low threshold (failure to respond to two drugs, one an atypical), above which clozapine treatment should be considered (National Institute for Clinical Excellence, 2002).

\section{Maintenance in the community}

It goes without saying that every effort should be made to avoid loss to follow up of people with schizophrenia living in the community. Individuals who are stable and adhere to medication regimens and have no residual difficulties requiring care may eventually be discharged to primary care, but such people may very much be in the minority.

Patients should be encouraged to participate in their own care. It is important to discuss medication with each patient, so that they feel involved in decision-making. It has been pointed out (Anonymous, 2004) that choice of atypical antipsychotic is not simple, with no consensus on differences in efficacy, and possibly inadequate information regarding differential side-effect profiles. Therefore, the preferences of patients, and their relatives and carers, may contribute to these decisions.

In maintenance treatment the simplest regimen of medication at the minimum effective dose is usually acceptable, ideally once daily. The only clinical benefit of using depot medication is knowing when a patient has defaulted, although this knowledge will usefully inform subsequent management.

\section{Side-effects}

Patients should always be observed for, and questioned specifically about, unwanted side-effects. 
These, particularly parkinsonism, akathisia, sexual dysfunction, sedation and weight gain, are a major cause of non-adherence. Side-effects should be managed by dose reduction (preferably), a change to a drug with less liability for that specific effect, or the addition of an appropriate antidote. However, in individuals with optimal mental states who adhere to their medication regimen, changing the antipsychotic is not a simple decision. The risks of significant loss of therapeutic response, the emergence of new problematic side-effects and the understandable anxiety of the patient and their carers must be balanced against the benefits accruing from side-effect management.

\section{Weight gain and diabetes}

Weight gain is particularly difficult to address, requiring as it does substantial and permanent dietary and lifestyle changes in a population not known for healthy living. Marked weight gain has been observed with atypical antipsychotics, although it is also a problem with the typicals. Histamine receptor affinity and dopamine affinity relative to 5- $\mathrm{HT}_{2}$ receptors both seem to be robust correlates of weight increase. Low pre-treatment body mass index, young age and female gender increase liability. There are some reports of an association between weight gain and clinical improvement (Russell \& Mackell, 2001).

It remains unclear how much weight gain contributes to type II diabetes and hyperlipidaemia. A large systematic review of weight gain ranked clozapine as presenting the highest risk of gain, followed by olanzapine, quetiapine, risperidone, sertindole, zotepine and amisulpride (Taylor \& McAskill, 2000). There is no known association yet with aripiprazole.

The World Health Organization has estimated that the worldwide prevalence of diabetes will more than double between 1995 and 2025 (Buse, 2002). People with schizophrenia are known to have a twoto threefold increased risk for type II diabetes. The strength of the association between antipsychotics and diabetes varies for individual medications, with the largest number of reports for chlorpromazine, clozapine and olanzapine (Henderson, 2002). It would seem prudent to monitor patients' weight, glucose and lipid levels, whatever antipsychotic they are prescribed.

\section{QTc interval}

Regarding QTc interval, prolongation greater than $500 \mathrm{~ms}$ is a risk factor for torsades de pointes, a potentially fatal ventricular arrhythmia, but is not the direct cause. Olanzapine, quetiapine and risperidone have not been associated with torsades de pointes; there are no data on amisulpride, zotepine and aripiprazole. It is recommended that antipsychotic drugs are not used in people for whom their benefit is marginal and that they should be used with caution in individuals with established cardiovascular disease.

\section{Social issues and quality of life}

Maintenance treatment should never be limited to medication issues. Patients and their carers always require support and assistance with many aspects of life: relationships within the family, planning for the future, employment, education, training, housing, benefits, social integration, all of which contribute to quality of life or lack of it. In this respect patients and carers are just like any other people, but they have special difficulties in fulfilling their needs. Although a purely psychiatric medical model cannot address such issues directly, it should be the overall long-term responsibility of the psychiatrist within the multidisciplinary (and possibly multiagency) team to make sure that unmet needs are recognised and resolved as far as possible by team members.

There are numerous voluntary agencies and charities (such as Rethink, Mind and SANE) that aim to assist people with mental illness and their carers. Furthermore, there are often local self-help and carers' groups which can provide valuable support outside the mainstream. Such bodies often welcome input from professionals in the form of education and advice, and they can make valuable comment on the strengths and shortcomings of services.

\section{Specific long-term problems Patients who do not get better}

The first step in managing patients who do not get better is to rule out obvious causes: diagnostic inaccuracy (for instance, unsuspected underlying physical disorder), substance misuse and nonadherence. High levels of stress within the family or care setting may also contribute. Some patients will be depressed and so not functioning optimally. Leaving aside these factors and the interventions needed to address them, it is important to specify in exactly what way the individual is failing to improve. Uncontrolled positive symptoms, unresolved negative symptoms and cognitive deterioration can all combine to compromise personal and social function. The first strategy to be implemented should be a medication review particularly to assess the current regimen and to identify drugs that helped in the past and unused options. Polypharmacy and 
chronic anticholinergic use, for instance, can detract from the mental state, as can side-effects such as oversedation and akathisia. Conventional antipsychotics are known to be depressogenic (Mortimer et al, 2003).

\section{Alternative and adjunctive drug treatment}

If no addressable issues are identified, as mentioned above the NICE guidance is to apply a much lower threshold for offering clozapine than has previously been the case in routine clinical practice (National Institute for Clinical Excellence, 2002). It is always a major step to start clozapine: although there is incontrovertible evidence that most treatment-resistant individuals benefit significantly to substantially, its side-effect profile, except for extrapyramidal phenomena, is particularly problematic. I have found that weight gain, drowsiness, myoclonus, gastrointestinal disturbances and worrying cardiac phenomena have led to more discontinuations than have neutropenia and non-response.

For individuals who cannot or will be unlikely to tolerate clozapine, or those who refuse to take it or prefer to try other options first, there are several possibilities (Box 3). Their evidence base does not compare with that for clozapine but they may be worth a try, although adjuvant drugs may have little effect on unresolved symptoms even when used appropriately (Buchanan et al, 2002). Recent reviews of antipsychotic polypharmacy and psychotropic adjunctive treatment came to opposing conclusions (Lerner et al, 2004; Stahl \& Grady, 2004).

Individuals with genuine problematic negative symptoms may benefit from the addition of a specific serotonin reuptake inhibitor (SSRI; Silver, 1998; Joffe et al, 1999), although these can be associated with exacerbation of positive symptoms and agitation. Low-dose amisulpride, other classes of antidepressant, glutamatergic drugs and dopaminergic agonists can also be tried (Mortimer, 2001).

\section{Box 3 Adjunctive medication}

- Positive symptoms: semisodium valproate (divalproex), lamotrigine

- Negative symptoms: low-dose amisulpride (monotherapy), SSRIs

- Symptoms in general: modafinil (preliminary evidence)

- Affective symptoms: lithium

- Depression: standard antidepressants

- Catatonia: lorazepam 6 mg daily, ECT

- Clozapine non-response: add amisulpride or risperidone
Semisodium valproate (divalproex) may be helpful in the management of poorly controlled positive symptoms in acute relapse: one small open study of semisodium valproate augmentation of haloperidol reported $45 \%$ fewer in-patient days in the augmented group (Wassef et al, 2001). Response to treatment was particularly noted for suspiciousness, hallucinations, unusual thought content (i.e. delusions) and emotional withdrawal. Effects on psychomotor agitation and aggression arising from temporal lobe pathophysiology have been proposed (Winterer \& Hermann, 2000). Another anticonvulsant, lamotrigine, has demonstrated efficacy in reducing positive symptoms in combination with clozapine (Tiihonen et al, 2003).

Adjunctive modafinil has been associated with generalised improvements in most patients in one small study (Rosenthal \& Bryant 2004).

Adjunctive lithium may benefit up to half of all patients (Siris, 1993). Predictors of response to lithium include affective symptoms, aggression and family history of affective disorder. Recent work has demonstrated that the response of individuals with schizophrenia who do not have prominent affective symptoms is marginal (Leucht et al, 2004). This is also true of patients treated with a combination of lithium and clozapine, although lithium's effect in increasing the neutrophil count may be very useful in such circumstances (Small et al, 2003). Further strategies for non-responding patients include adding standard antidepressant treatment for depression (not forgetting psychological therapy) and adding benzodiazepines such as clonazepam for agitation and anxiety. The treatments of choice for catatonia are lorazepam $6 \mathrm{mg}$ daily or electroconvulsive therapy (ECT; Rosebush 2004).

\section{Cognitive-behavioural therapy and cognitive remediation}

Engaging the patient in reinforceable activity can minimise negative symptoms by eliciting cognitive, affective and behavioural responses. However, it is likely that this must be a permanent strategy. Cognitive-behavioural therapy may also be worth pursuing (Sensky et al, 2000). It is for positive symptoms, particularly reality distortion (hallucinations and delusions), that such therapy is most frequently indicated. A recent meta-analysis concluded that cognitive-behavioural therapy resulted in important sustained improvements in mental state and that it could be useful for people with treatment-resistant symptoms.

Cognitive remediation is a psychological treatment in which patients practise neuropsychological tasks, in the expectation that proficiency will generalise to a variety of real-life situations, 
improving social outcome and reducing symptomatology. Leaving aside the scarcity of such treatment opportunities, there is controversy regarding its effectiveness: a recent meta-analysis, which also scrutinised social skills training, failed to find reliable benefits and did not recommend either treatment (Pilling et al, 2002b).

Awakenings

Attention has been drawn to the 'awakening' phenomenon (Weiden et al, 1996). Patients who have been out of touch with reality for substantial periods can make a pronounced recovery, usually but not exclusively during successful clozapine treatment. Unfortunately, such a return to relatively normal mental health is often accompanied by understandable distress regarding their illness, its implications and the effective loss of sometimes years of previous life, accompanied by marked apprehension about what the future holds and considerable reluctance to disengage from the sick role. Such events require early recognition, careful psychological support, and a gentle and realistic approach to reintegration of the patient with family and society. In addition, gratifying recovery may lead the treatment team to become overoptimistic about how much responsibility and choice the patient can manage. Unfortunately, some patients withdraw from the recovery process, becoming non-adherent with medication regimens and reverting to psychotic behaviour. Sometimes the only option is to respect their choice and make long-term provision for continuing care.

\section{Disengagement from services \\ Assertive outreach}

Assertive outreach services were and continue to be developed in order to manage patients who consistently default from follow-up and are likely to stop their medication as soon as possible, relapse into acute psychosis and require readmission repeatedly and inevitably. The assertive outreach model's most important tenet is that no staff member will have a case-load of more than ten clients. Furthermore, the team is multidisciplinary, ideally multi-agency, so that clients can be offered assistance for any reasonable unmet need, thus fostering engagement with the service. Contact may mostly take place outside standard mental health facilities: team members should be able to support key family members, carers and staff of other mental health, social services and housing agencies.

Assertive outreach is particularly useful for managing risk in the community for the most difficult individuals with schizophrenia: those who are dually diagnosed with substance misuse or comorbid personality disorder, are non-adherent, lack insight, are offenders, and sometimes all of these things. Patients are not usually discharged from assertive outreach services, which try to offer consistent care over longer periods and to provide more intense support than can be accessed from a community mental health team. The risks that can be managed by assertive outreach include that of deliberate self-harm as well as harm to others. Community supervision under Section 25A of the Mental Health (Patients in the Community) Act 1995 may be a particularly apposite means of encouraging engagement, supervising taking of medication and enabling acute crises to be resolved.

It is important to be realistic about the outcomes for patients of assertive outreach: it should not be seen as a way of preventing admissions, for instance, or increasing patient 'throughput', but as a way of reducing overall harm. Furthermore, the team must be sufficiently confident to recognise when it is not realistic to manage the risk presented by patients in the community, and to refer to more appropriate services.

I have found considerable overlap between the groups of patients under assertive outreach services, those in semi-supported and residential care, those in rehabilitation units, and those who are eventually placed in psychiatric nursing and low secure care facilities. For patients presenting less risk but more disability, assertive outreach may become community rehabilitation: the delivery of psychosocial interventions to promote social integration and maximise personal function, through the use of ordinary community resources whenever possible.

\section{Substance misuse}

Comorbidity of substance use disorders and schizophrenia is associated with a greater risk of serious illness complications and poorer outcome, including suicide. About half of people with schizophrenia have a history of past or current substance misuse (Swafford et al, 2000). Integrated treatment services demonstrate the strongest evidence for effective management (Tsuang \& Fong, 2004).

Drake et al (2001) describe the components of successful dual diagnosis services. These include a comprehensive, long-term, staged approach to recovery; assertive outreach; motivational interventions; providing clients with help to acquire skills and with supports to manage both illnesses and pursue functional goals; and cultural sensitivity and competence. Drake et al concluded that high-quality services were rare. 
It is important to find out about the individual's substance misuse and to offer access to substance misuse treatment services, including voluntary agencies. Motivation to change is often lacking initially, but may eventually be forthcoming. In the meantime, management on the lines of harm reduction is indicated, alongside appropriate support for carers. Some authorities recommend specific pharmacotherapy: clozapine and atypical antipsychotic drugs for psychosis together with, for example, buproprion for smoking cessation (Noordsy \& Green, 2003).

\section{Knowing when to stop trying}

Recovery models of outcome in schizophrenia are a laudable aspiration, but they may not be feasible for prevalent as opposed to incident cases. Essentially, the patient's environment and the carers within it must compensate for loss of personal and social function unresponsive to pharmacological and psychological strategies (Box 4). An in-patient rehabilitation unit is the ideal setting in which to evaluate these deficits and their treatability, and to restore loss of function as far as possible before appropriate community placement.

At the mildest end of the spectrum, a patient may need to live in sheltered or semi-supported housing, where a package of care can be delivered. This might include help with housework, budgeting, shopping and meal preparation, transport to and encouragement to undertake occupational, social and leisure pursuits, and monitoring of treatment adherence. Such arrangements are perhaps the best solution for individuals who persistently state they 'want a flat' but who have repeatedly failed to cope with living independently.

Individuals unable to remain in remission in such a care environment may need residential hostel accommodation, living in a group situation with meals and laundry provided. Stability is important here: some facilities focus on further rehabilitation with the objective of 'moving on'. It is prudent to advise the staff, and possibly the patient, if this is

\section{Box 4 Resources for severely ill individuals}

- In-patient rehabilitation units

- Assertive outreach services

- Home treatment teams

- Semi-supported accommodation

- Residential care homes

- Psychiatric nursing homes

- Low secure care units anticipated to be unrealistic. Such pressures can make relapse more likely, and with it loss of the placement.

A small group of individuals are chronically psychotic and unamenable to further treatment, with disturbed behaviour and major personal and social incapacity, and these people require psychiatric nursing. Continuing care facilities are scarce, often privately run and difficult to access because of funding issues. To these disadvantages may be added considerable distances from the patients' locality and family members, and lack of choice, which may dictate suboptimal placement because the alternative is permanent blocking of a rehabilitation or acute bed.

An even smaller group require low secure care at least in the medium term and possibly on a permanent basis. Every effort should be made to prevent schizophrenia from reaching this level of severity. Unfortunately, however, some individuals will prove untreatable in open nursing facilities because of their unsafe behaviour. This may include extremely bizarre behaviour such as hyperkinesia (catatonic agitation), suicidality, violence, absconding, hazardous substance misuse and the risk of acting on dangerous delusions. Fortunately, it is not uncommon to find that a lengthy period of treatment in a local psychiatric intensive care unit, in low secure conditions, is effective and can obviate the need for longer-term secure care if follow-up is assertive.

\section{Conclusions}

The long-term treatment of people with schizophrenia should have been greatly facilitated by the advent of better tolerated and probably more effective antipsychotic drugs, the relaunch of clozapine, and substantial advances in the development of psychological therapies, psychosocial interventions and early treatment initiatives. However, experience suggests that outcomes remain dubious for many individuals: the deinstitutionalisation of care and the rising tide of substance misuse have perhaps conspired to minimise treatment adherence and engagement. Services are faced every day with the inevitable conflict between the patient's free will and the service's responsibility to society, in parallel with the conflict between the rights of the patient and the rights of the community. To these difficulties may be added a chronic shortage of senior medical expertise, a dearth of resources for the implementation of psychosocial interventions, and the failure of the public sector to accommodate the worst affected patients itself. Meanwhile, the pathophysiology of schizophrenia remains essentially unknown, despite extensive research efforts. 
It would be naïve to expect the advent of early intervention services to obviate further accumulation of damaged individuals and the necessity to care for them. Most clinicians would agree, however, that prompt diagnosis, early treatment and psychosocial interventions represent the best hope of reducing the overall burden of long-term schizophrenia for patients, families and services in the future.

\section{References and related articles}

Abi-Dargham, A. (2002) Recent evidence for dopamine abnormalities in schizophrenia. European Psychiatry, 17, 341-347.

Anonymous (2004) Atypical antipsychotics in schizophrenia. Drugs and Therapeutics Bulletin (August), 57-60.

Arvanitis, L. A., Miller, B. G. \& the Seroquel Trial Study Group (1997) Multiple fixed doses of 'Seroquel' (quetiapine) in patients with acute exacerbation of schizophrenia: a comparison with haloperidol and placebo. Biological Psychiatry, 42, 233-246.

Barnas, C., Stuppack, C. H., Miller, C., et al (1992) Zotepine in the treatment of schizophrenic patients with prevailingly negative symptoms, a double blind trial versus haloperidol. International Clinical Psychopharmacology, 7, 23-27.

Bowles, T. M. \& Levin, G. M. (2003) Aripiprazole: a new atypical antipsychotic drug. Annals of Pharmacotherapy, 37, 687-694.

Buchanan, R. W., Kreyenbuhl, J., Zito, J. M., et al (2002) Relationship of the use of adjunctive pharmacological agents to symptoms and level of function in schizophrenia. American Journal of Psychiatry, 159, 1035-1043.

Buse, J. B. (2002) Metabolic side-effects of antipsychotics: focus on hyperglycaemia and diabetes. Journal of Clinical Psychiatry, 63 (suppl. 4), 37-41.

Cowen, P. J. (2005) New drugs, old problems. Advances in Psychiatric Treatment, 11, 19-27.

Czekalla, J., Kollach-Walker, S. \& Beasley, C. M. (2001) Cardiac safety parameters of olanzapine: comparison with other atypical and typical antipsychotics. Journal of Clinical Psychiatry, 62, (suppl. 2), 35-40.

Davis, J. M. (1975) Overview: maintenance therapy in psychiatry. 1: Schizophrenia. American Journal of Psychiatry, 132, 1237-1245.

Davis, J. M. \& Casper, R. (1977) Antipsychotic drugs: clinical pharmacology and therapeutic use. Drugs, 14, 260-282.

Drake, R. E., Essock, S. M., Shaner, A., et al (2001) Implementing dual diagnosis services for clients with severe mental illness. Psychiatric Services, 52, 469-476.

Edwards, J. G. (2005) Newer $v$. older antidepressants in longterm pharmacotherapy. Revisiting... Prevention of relapse and recurrence of depression. Advances in Psychiatric Treatment, 11, 184-194.

Gournay, K. (2005) The changing face of psychiatric nursing. Advances in Psychiatric Treatment, 11, 6-11.

Green, B. (2000) Focus on risperidone. Current Medical Research and Opinion, 16, 57-65.

Henderson, D. C. (2002) Atypical antipsychotic-induced diabetes mellitus: how strong is the evidence? CNS Drugs, 16, 77-89.

Hunt, G. E., Bergen, J. \& Bashir, M. (2002) Medication compliance and comorbid substance abuse in schizophrenia: impact on community survival 4 years after a relapse. Schizophrenia Research, 54, 253-264.

Hwang, T. J., Lin, S. K. \& Lin, H.-N. (2001) Efficacy and safety of zotepine for the treatment of Taiwanese schizophrenic patients: a double-blind comparison with haloperidol. Journal of the Formosan Medical Association, 100, 811-816.

Joffe, G., Appelberg, B. \& Rimon, R. (1999) Adjunctive nefazodone in neuroleptic-treated schizophrenic patients with predominantly negative symptoms: an open prospective pilot study. International Clinical Psychopharmacology, 14, 233-238.

Kerwin, R. W. \& Bolonna, A. (2005) Management of clozapineresistant schizophrenia. Advances in Psychiatric Treatment, 11, 101-106

Kopelowicz, A., Zarate, R., Tripodis, K., et al (2000) Differential efficacy of olanzapine for deficit and nondeficit negative symptoms in schizophrenia. American Journal of Psychiatry, 157, 987-993.

Lacro, J. P., Dunn, L. B., Dolder, C. R., et al (2002) Prevalence of and risk factors for medication nonadherence in patients with schizophrenia: a comprehensive review of recent literature. Journal of Clinical Psychiatry, 63, 892-909.

Lane, H. Y., Chiu, W. C., Chou, J. C., et al (2000) Risperidone in acutely exacerbated schizophrenia: dosing strategies and plasma levels. Journal of Clinical Psychiatry, 61, 209-214.

Lerner, V., Libov, I., Kotler, M., et al (2004) Combination of atypical antipsychotic medication in the management of treatment resistant schizophrenia and schizoaffective disorder. Progress in Neuro-Psychopharmacology and Biological Psychiatry, 28, 89-98.

Leucht, S., Pitschel-Walz, G., Abraham, D., et al (1999) Efficacy and extrapyramidal side-effects of the new antipsychotics olanzapine, quetiapine, risperidone, and sertindole compared to conventional antipsychotics and placebo. A meta-analysis of randomized controlled trials. Schizophrenia Research, 35, 51-68.

Leucht, S., Kissling, W. \& McGrath, J. (2004) Lithium for schizophrenia revisited: a systematic review and metaanalysis of randomized controlled trials. Journal of Clinical Psychiatry, 65, 177-186.

Marder, S. R. \& Meibach, R. C. (1994) Risperidone in the treatment of schizophrenia. American Journal of Psychiatry, 151, 825-835.

Margolese, H. C., Malchy, L., Negrete, J. C., et al (2002) Drug and alcohol use among patients with schizophrenia and related psychosis: levels and consequences. Schizophrenia Research, 67, 157-166.

Moller, H.-J. (2000) Amisulpride: a review of its efficacy in schizophrenia. Acta Psychiatrica Scandinavica, Supplementum, 400, $17-22$.

Mortimer, A. (1997) Treatment of the patient with long-term schizophrenia. Advances in Psychiatric Treatment, 3, 339346.

Mortimer, A. M. (2001) Pharmacological treatment approaches. In Managing Negative Symptoms of Schizophrenia (eds A. Mortimer \& S. Spence), pp. 47-59. London: Science Press.

Mortimer, A. M., Martin, C., Wheeler, J. A., et al (2003) Antipsychotic prescription in unipolar depression. 2: Withdrawing antipsychotics in unipolar, non-psychotic patients. Journal of Clinical Psychiatry, 64, 668-672.

Munro, J., O'Sullivan, D., Andrews, C., et al (1999) Beyond pharmacovigilance: active monitoring of 2,760 patients receiving clozapine in the UK and Ireland. British Journal of Psychiatry, 175, 576-580.

National Institute for Clinical Excellence (2002) Guidance on the Use of Newer (Atypical) Antipsychotic Drugs for the Treatment of Schizophrenia (Technology Appraisal Guidance no. 43). London: NICE.

Noordsy, D. L. \& Green, A. I. (2003) Pharmacotherapy for schizophrenia and co-occurring substance use disorders. Current Psychiatry Reports, 5, 340-346.

Peuskens, J. \& Link, C. G. G. (1997) A comparison of quetiapine and chlorpromazine in the treatment of schizophrenia. Acta Psychiatrica Scandanavica, 96, 265-273.

Pilling, S., Bebbington, P., Kuipers, E., et al (2002a) Psychological treatments in schizophrenia: I. Meta-analysis of family intervention and cognitive behaviour therapy. Psychological Medicine, 32, 763-782.

Pilling, S., Bebbington, P., Kuipers, E., et al (2002b) Psychological treatments in schizophrenia: II. Meta-analyses of randomized controlled trials of social skills training and cognitive remediation. Psychological Medicine, 32, 783-791. 
Raniwalla, J., Tweed, J. A., Dollfus, S., et al (1996) A comparison of an atypical (zotepine) and classical (haloperidol) antipsychotic in patients with acute exacerbation of schizophrenia. Schizophrenia Research, 18, 133.

Robinson, D. G., Woerner, M. G., McMeniman, M., et al (2004) Symptomatic and functional recovery from a first episode of schizophrenia or schizoaffective disorder. American Journal of Psychiatry, 161, 473-479.

Rosebush, P. I. (2004) Treatment of catatonia. In Catatonia: from Psychopathology to Neurobiology (eds S. N. Caroff, S. C. Mann, A. Francis, et al), pp. 141-150. Washington, DC: American Psychiatric Publishing.

Rosenthal, M. H. \& Bryant, S. L. (2004) Benefits of adjunct modafinil in an open-label, pilot study in patients with schizophrenia. Clinical Neuropharmacology, 27, 38-43.

Russell, J. M. \& Mackell, J. A. (2001) Bodyweight gain associated with atypical antipsychotics: epidemiology and therapeutic implications. CNS Drugs, 15, 537-551.

Seivewright, N., McMahon, C. \& Egleston, P. (2005) Stimulant use still going strong. Revisiting... Misuse of amphetamines and related drugs. Advances in Psychiatric Treatment, 11, 262-269.

Sensky, T., Turkington, D., Kingdon, D., et al (2000) A randomized controlled trial of cognitive-behavioural therapy for persistent symptoms in schizophrenia resistant to medication. Archives of General Psychiatry, 57, 165-172.

Silver, H. S. N. (1998) Augmentation with fluvoxamine but not maprotiline improves negative symptoms in treated schizophrenia: evidence for a specific serotonergic effect from a double-blind study. Journal of Clinical Psychopharmacology, 18, 208-211.

Singh, S. P. \& Fisher, H. L. (2005) Early intervention in psychosis: obstacles and opportunities. Advances in Psychiatric Treatment, 11, 71-78.

Siris, S. G. (1993) Adjunctive medication in the maintenance treatment of schizophrenia and its conceptual implications. British Journal of Psychiatry, 163 (suppl. 22), 66-78.

Small, J. G., Clapper, M. H., Malloy, F. W., et al (2003) Tolerability and efficacy of clozapine combined with lithium in schizophrenia and schizoaffective disorder. Journal of Clinical Psychopharmacology, 23, 223-228.

Song, F. (1997) Risperidone in the treatment of schizophrenia: a meta-analysis of randomised controlled trials. Journal of Psychopharmacology, 11, 65-71.

Stahl, S. M. \& Grady, M. M. (2004) A critical review of atypical antipsychotic utilization: comparing monotherapy with polypharmacy and augmentation. Current Medicinal Chemistry - Central Nervous System Agents, 11, 313-327.

Swafford, C. D., Scheller-Gilkey, G., Miller, A. H., et al (2000) Double jeopardy: schizophrenia and substance abuse. American Journal of Drug and Alcohol Abuse, 26, 343-353.

Taylor, D. M. \& McAskill, R. (2000) Atypical antipsychotics and weight gain - a systematic review. Acta Psychiatrica Scandinavica, 101, 416-432.

Tiihonen, J., Hallikainen, T., Ryynanen, O.-P., et al (2003) Lamotrigine in treatment-resistant schizophrenia: a randomized placebo-controlled crossover trial. Biological Psychiatry, 54, 1241-1248.

Tsuang, J. \& Fong, T. W. (2004) Treatment of patients with schizophrenia and substance abuse disorders. Current Pharmaceutical Design, 10, 2249-2261.

Wassef, A. A., Hafiz, N. G., Hampton, D., et al (2001) Divalproex sodium augmentation of haloperidol in hospitalized patients with schizophrenia: clinical and economic implications. Journal of Clinical Psychopharmacology, 21, 21-26.

Weiden, P., Aquila, A. \& Standard, J. (1996) Atypical antipsychotic drugs and long-term outcome in schizophrenia. Journal of Clinical Psychiatry, 57 (suppl. 11), 5360

Winterer, G. \& Hermann, W. M. (2000) Valproate and the symptomatic treatment of schizophrenia spectrum patients. Pharmacopsychiatry, 33, 182-188.

\section{MCQs}

1 Atypical antipsychotics:

a are at least as efficacious for positive symptoms as conventional antipsychotic drugs

b are all associated with fewer extrapyramidal symptoms than conventional antipsychotic drugs

c are not associated with hyperprolactinaemia

$\mathrm{d}$ have as a class been positively associated with torsades de pointes

e overall cause more hyperglycaemia than conventional antipsychotics.

2 The following may be useful when added to antipsychotic monotherapy:

a semisodium valproate

b benperidol

c phenobarbitone

d fluoxetine

e modafinil.

3 The following treatments have been recommended by recent meta-analyses:

a cognitive remediation

b family interventions

c essential polyunsaturated fatty acids

d cognitive-behavioural therapy

e social skills training.

4 Fidelity to the assertive outreach model is demonstrated by services which:

a have fewer than ten clients per staff member

b frequently transfer patients to generic community services

c are multidisciplinary and multi-agency in their staff $\operatorname{mix}$

d focus on patients who disengage from services

e carry out the majority of client work in the out-patient clinic.

5 Regarding patients with schizophrenia:

a $30 \%$ maintain a full recovery from first episode 5 years later

b less than $20 \%$ engage in substance misuse

c over $80 \%$ are non-adherent in any treatment setting

d cognitive impairment is rare

e reasons for discontinuation of clozapine treatment are limited to neutropenia.

\section{MCQ answers}

\begin{tabular}{|c|c|c|c|}
\hline 1 & 2 & 3 & 4 \\
\hline a $\mathrm{T}$ & a $\mathrm{T}$ & a $F$ & a $\mathrm{T}$ \\
\hline $\mathrm{T}$ & $b F$ & $\mathrm{~b} T$ & b F \\
\hline $\mathrm{F}$ & C F & C F & c $\mathrm{T}$ \\
\hline $\mathrm{F}$ & $\mathrm{d} T$ & d $\mathrm{T}$ & $\mathrm{d} \mathrm{T}$ \\
\hline $\mathrm{F}$ & e $\mathrm{T}$ & e $F$ & e $F$ \\
\hline
\end{tabular}

